\title{
Analysis of high velocity impacts of quasibrittle material fragments with a Hopkinson bar
}

\author{
J. M. Rodríguez-Sereno ${ }^{1}$, J. Pernas-Sánchez ${ }^{1}$, J.A. Artero-Guerrero ${ }^{1}$, J. López Puente ${ }^{1}$ \\ ${ }^{1}$ Department of Continuum Mechanics and Structural Analysis, University Carlos III Madrid
}

Avda. De la Universidad, 30, 28911 Leganés, Spain

\begin{abstract}
In this work it has been characterized the impact behaviour of quasibrittle fragments against a Hopkinson bar in a wide range of impact velocities $(70-180 \mathrm{~m} / \mathrm{s})$. Hopkinson bar is designed to measure the impact forces thanks to a proper instrumentation. In this case, semiconductor strain gauges, which sensibility is higher than common strain gauges, are used. Two material are studied to widen the range of applicability of the experimental facility: PMMA and a woven composite material. Moreover, two high speed cameras (Photron SA-Z) have been used to obtain the displacement and strain field of the fragment impactor using 3D Digital Image Correlation (DIC). A special technique has been used to print the speckle in the fragment based on previous works. The developed experimental methodology has shown the different failure modes that may happen in the fracture process when the quasibrittle fragments acts as the impactor providing a better understanding of its physical behaviour.
\end{abstract}

\section{Introduction}

The aeronautic industry is an engineering field in which aircraft designers are looking for new solutions for the structure and the engines to decrease the fuel consumption. The fuel cost is one of the main components of the airlines operating costs, and its reduction is crucial for the transport industry. In addition, it is important to highlight that the air transport accounts the $2 \%$ of all green-house gases emitted to the atmosphere and the developing of new technologies will diminish the contamination footprint of this industry (in addition a 60\% increment in traffic is expected for the next two decades [1]).

Counter-rotating open rotor are currently being investigated within the frame of reducing fuel consumption and noise [2]. A potential problem that should be taken in account is the possibility of the failure of one of these blades, which may impact against the fuselage [3]. These blades may be designed and manufactured with composite materials, and therefore it will be needed to perform investigations about the behaviour of a CFRP blade impacting against an aircraft structure. In the literature it can be found many different researches about the behaviour of composite when they are subjected to impacts, but there only a few [3] that study the case of a composite fragment acting as an impactor.

In this work, it has been developed an experimental procedure to analyse the behaviour of the impact event of a quasibrittle fragment. It has designed a Hopkinson bar, based in the work of A. Pereira et al. [1] to obtain the impact forces in the event. Two material are studied to widen the range of applicability of the experimental facility: PMMA and a woven composite material. Moreover, two high speed cameras (Photron SA-Z) have been used to obtain the displacement and strain field of the fragment impactor using 3D Digital Image Correlation (DIC). In the following sections, the results obtained from the impact of fragments made of PMMA and CFRP will be shown.

\section{Experimental procedure}

In this section, the experimental methodology developed for analysing the behaviour of quasibrittle fragment impacts is explained. 


\subsection{Materials and Tests}

The induced impact force against the Hopkinson Bar is studied using two different materials: Poly Methacrylate Methil Amide (PMMA) and Carbon Fibre Reinforced Polymer (CFRP) composite. In this case the composite material is made of a plain woven named AGP193-PW, composed of 8552 Epoxy Matrix and AS4 carbon fibres. The size for both impactors is (in millimetres) 42x100x4 (with 20 plies in the case of the woven composite fragments $[0]_{20}$ ).

\subsection{Experimental Set Up}

The gas gun has a squared inner area that avoid the rotation of the fragment during the acceleration. As aforementioned, the impacts are performed in a range of velocities between $70 \mathrm{~m} / \mathrm{s}$ and $180 \mathrm{~m} / \mathrm{s}$. This experimental device uses compressed air at pressures up to 6 bar, to impel the composite fragment through a $6 \mathrm{~m}$ long barrel [4]. To match the geometry of the fragment to the inner frontal area of the cannon a 3D printed PLA sabot has been designed.

The Hopkinson bar is designed to measure the impact forces in the dynamic event. Due to the differences in the mechanical behaviour of the impacted fragments, two different Hopkinson bars are used. For PMMA projectiles it has been used an Aluminium Alloy 2011 with a diameter of 50mm and length of $3 \mathrm{~m}$. For composite impactor, a Toolox 44 steel bar has been used, with a diameter of $51 \mathrm{~mm}$ and length of $3 \mathrm{~m}$. The high yield stress of Toolox 44 steel avoid the yielding of the bar during the composite fragment impact process, while the Aluminium bar allows to have a better sensitivity for PMMA impacts thanks to its smaller stiffness. In order to achieve an alignment between the gas cannon and the Hopkinson Bar, an iron structure is designed and performed (Fig1). The bar can move longitudinal with a negligible friction thanks to the roller presented in the structure.

Semiconductor Strain gauges have been located to 250 millimetres from the impact face in order to obtain the strain pulse of the impact. As a result, this experimental test developed allows obtaining the impact force assuming a homogeneous compressive wave. Also, the momentum induced by the fragment can be computed. Moreover, two high speed cameras (Photron SA-Z) have been used to obtain the displacement and strain field of the fragment impactor using 3D Digital Image Correlation (DIC) with the commercial software VIC3-D. The acquisition rate of the two Photron SA-Z cameras was set to 160,000 frames per second (fps) with a shutter speed of $800,000 \mathrm{1} / \mathrm{s}$. The resolution has been set to $128 \times 512$ pixel $^{2}$ that provide a resolution of more than $5 \mathrm{px} / \mathrm{mm}$. Additionally an appropriate lighting system has been used to ensure the obtention of bright images.

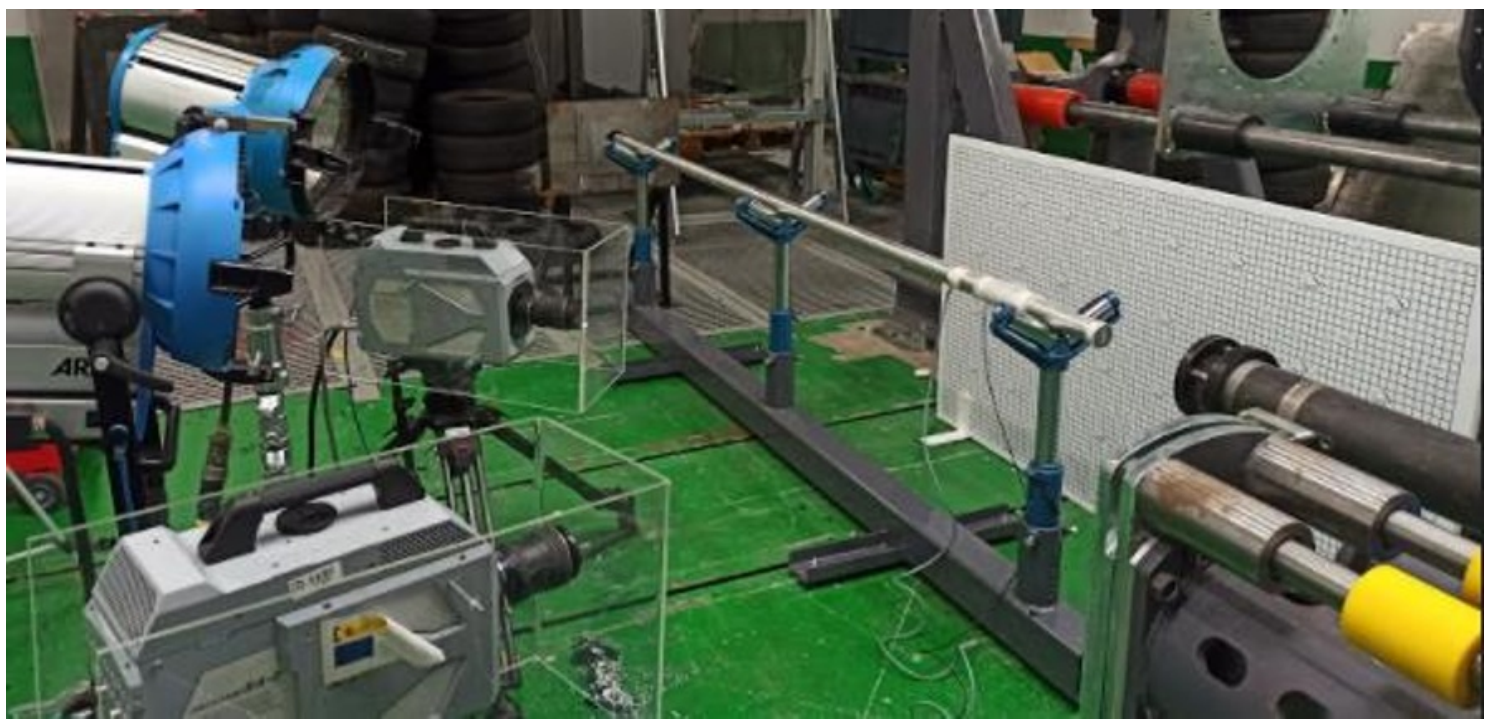

Fig. 1. Experimental Set Up 


\subsection{Speckle performed for Digital Image Correlation}

The induced impact force is a consequence of the mechanical behaviour of the fragments in the process of impact and the inertia forces. The mechanical behaviour may change due to the increment of the strain rate. It is demonstrated how an increment of the strain rate make the mechanical properties rise, developing in a stiffer and stronger material [5], [6].

In this work, an important parameter to describe the mechanical behaviour of the different materials in the impacts at different initial velocities, is the strain field along the specimen. These strains are measured using two high speed cameras (Photron SA-Z) and the VIC-3D software. The digital image correlation (DIC) technique is a simple way to obtain the strain field of the global specimen surface (Fig2). The DIC could be implemented using several techniques such as stamps or airbrush painting [6]. In this work, the water-slide paper technique developed by Quino et al. [7] has been chosen to ensure repeatability in all the experiments.
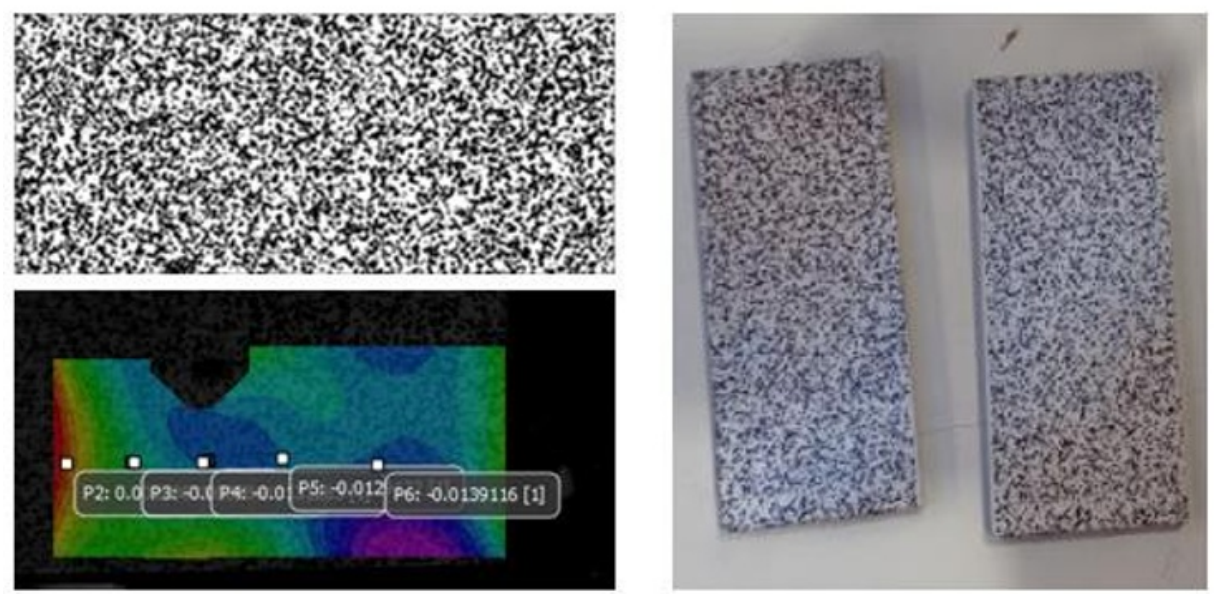

Fig. 2. DIC pattern using water-slide paper technique and the PMMA strain field

\section{Experimental Results}

In this section, the impact force and the strain field are analysed for similar kinetic energy impacts. Moreover, the high-speed video allows to compare and understand the different damage patterns induced.

\subsection{PMMA fragment impact results $v=116 \mathrm{~m} / \mathrm{s}$}

Figure 3 shows the strains measured in the specimen using the VIC3D software and the impact force induced in the Hopkinson Bar, obtained using the semiconductor strain gages, which allows to measure the compressive strain along the experiment.

Strain values are obtained using 5 different points among the specimen (see Figure 2). Knowing the distance of the points it can be calculated a stress wave velocity of $1760 \mathrm{~m} / \mathrm{s}$. The whole strain field of the PMMA fragment before the failure can be seen in Figure 4.

If a first approximation of the stress wave speed value is obtained with $C_{P M M A}=\sqrt{\frac{E_{P M M A}}{\rho_{P M M A}}}=1581 \mathrm{~m} / \mathrm{s}$ (where $\rho_{P M M A}$ and $E_{P M M A}$ are the density and stiffness of the PMMA respectively) it could be stated that the $3 \mathrm{D}-\mathrm{DIC}$ measures accurately the stress wave propagation. 

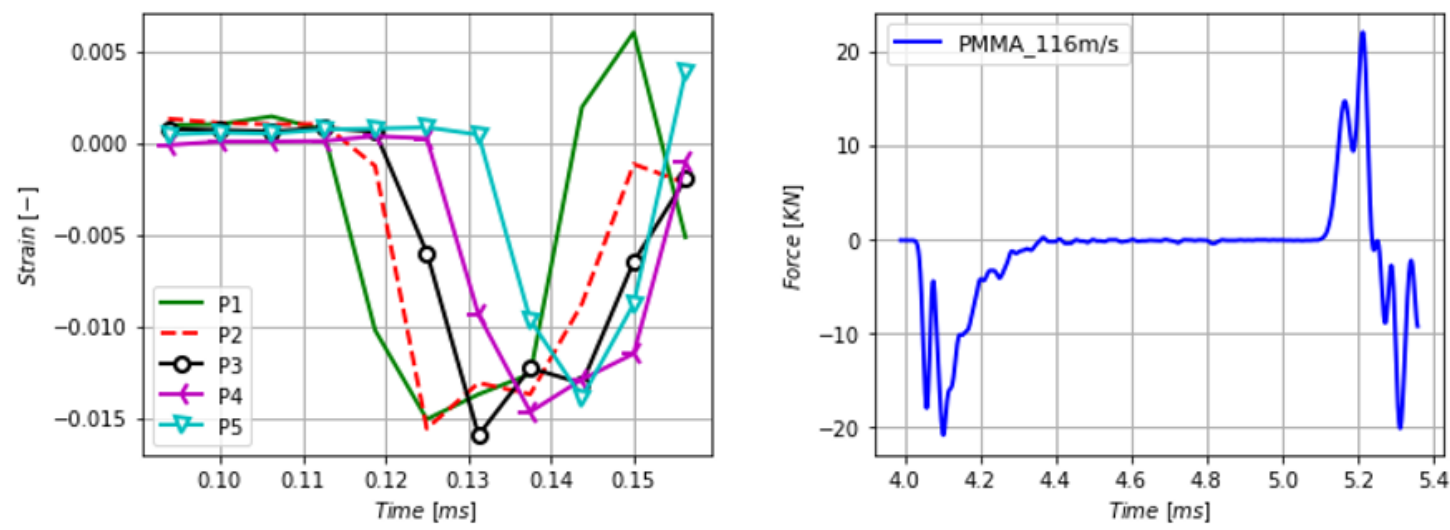

Fig. 3. a) PMMA Compressive strains at different points in the strain field and b) Impact Force in the Hopkinson Bar with the PMMA fragment launched at $116 \mathrm{~m} / \mathrm{s}$

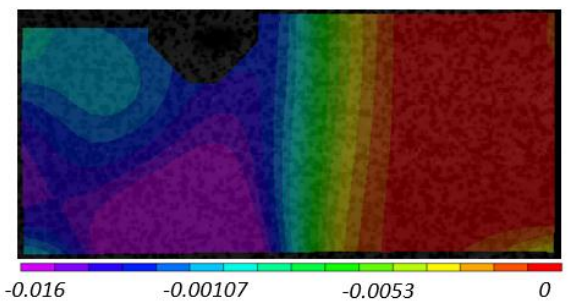

Fig. 4. Strain field of PMMA fragment launched at $116 \mathrm{~m} / \mathrm{s}$

Figure $3 \mathrm{~b}$ shows a first compressive pulse induced by the impact with a maximum of $20.62 \mathrm{kN}$ and a pulse duration $0.3 \mathrm{~ms}$. A second tensile pulse can be seen about $1 \mathrm{~ms}$ after produced by the reflection of the pulse at its end. Finally, the impulse is calculated performing the integral of the force, obtaining a value of $2.1 \mathrm{~N} \cdot \mathrm{s}$.

Since the theoretical impulse is $2.32 \mathrm{~N} \cdot \mathrm{s}$, it can be considered that experimental Hopkinson Bar data is valid. PMMA is a quasibrittle material whose failure mode at this impact condition is the total breakage, and therefore almost all impulse is transmitted to the bar during the impact. In the Figure 5 it can be observed the strain distribution during the impact, and how the compression pulse is almost flat (along the width of the projectile) in the specimen, showing the appropriate orientation of the impact. As it is shown in Figure 6, the damage pattern is the completely breakage of the specimen. The maximum compressive strain observed in the specimen is $1.5 \%$. The fragment reaches that value in a period of $0.01 \mathrm{~ms}$ (Fig 5).

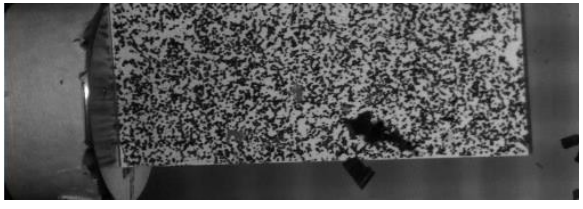

$t=0 \mathrm{~ms}$

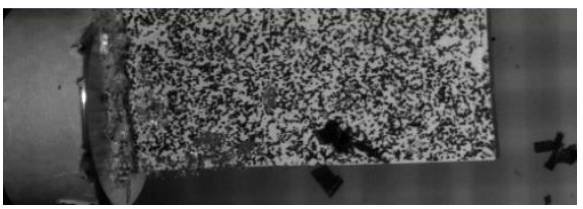

$t=0.05 \mathrm{~ms}$

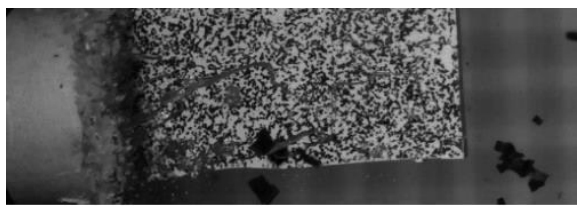

$t=0.2 \mathrm{~ms}$

Fig. 5. Impact experiment of PMMA fragment launched at $116 \mathrm{~m} / \mathrm{s}$

\subsection{CFRP fragment impact results $v=113 \mathrm{~m} / \mathrm{s}$}

In this section an analysis of the impact process for the woven composite specimen is performed. The impact analyzed was performed at $113 \mathrm{~m} / \mathrm{s}$, which is the test with a closer kinetic energy to the PMMA case from the previous section.

Figure 6 shows the strain field obtained using the VIC-3D software. The Photron SA-Z parameters are the same performed in the PMMA experiment, described in the previous section. Since the damage failure mode is different, and the composite specimen does not totally break like the polymer, it can be observed how the compressive pulse evolves. Two compressive pulses before the breakage can be observed, reaching a value of compressive strain of $1.1 \%$ in a period of $0.017 \mathrm{~ms}$. 
Moreover, the Figure 6 shown the impact force induced obtained thanks to the semiconductor strain gages. Compared to the PMMA strain pulse, the composite specimen applies an impact force almost 6 times higher (concretely, it reaches a value of $112 \mathrm{KN}$ ) and consequently the impact duration is much smaller.
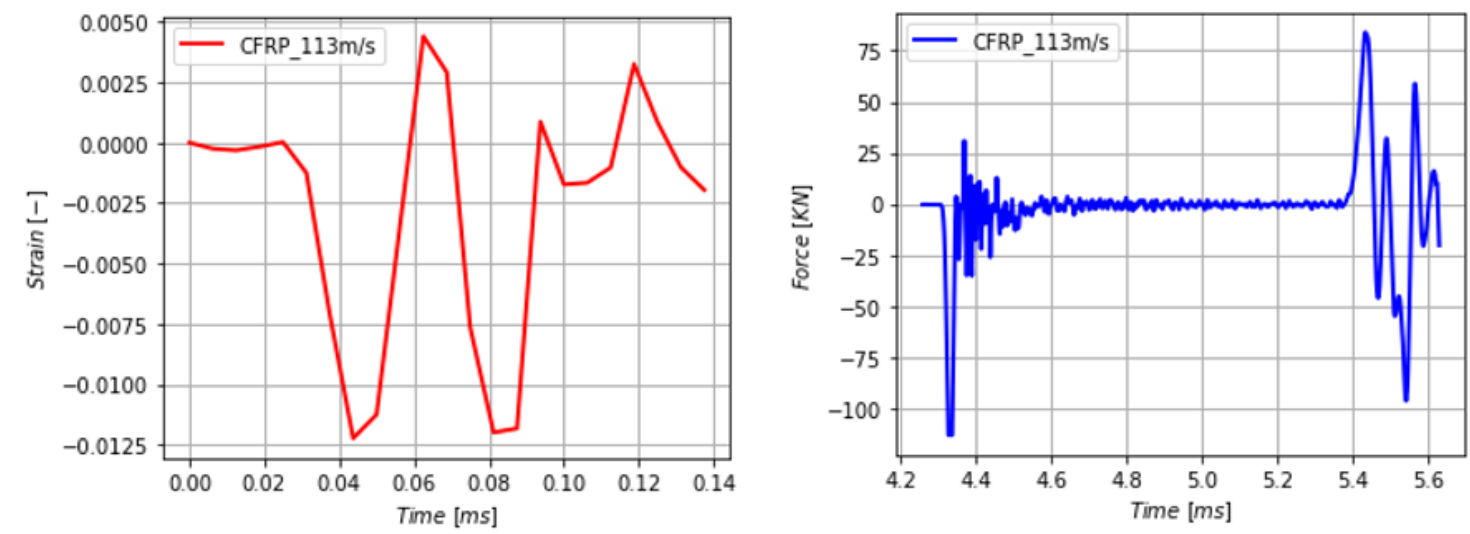

Fig. 6. a) CFRP compressive strain field and b) Impact force in the Hopkinson Bar with the CFRP fragment launched at $113 \mathrm{~m} / \mathrm{s}$

The Figure 7 shows the strain field analysed thanks to the VIC-3D software in the CFRP fragment. It can be observed how the compressive front pulse is not completely perpendicular to the edge of the fragment. Basing on the ratio between the impact velocity and the compressive pulse velocity (which is closer to 50) and the angle of the compressive front pulse measured thanks to the software, it can be concluded that the loss of perpendicularity in the impact is less than 1 degree.

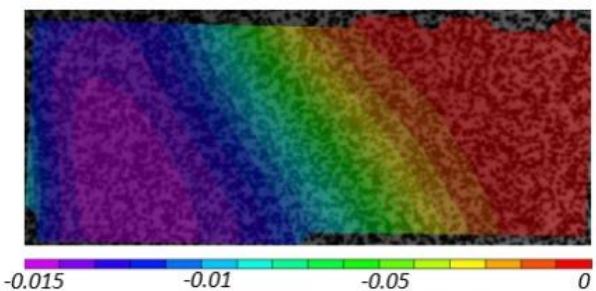

Fig. 7. Strain field of CFRP launched at $113 \mathrm{~m} / \mathrm{s}$

The Figure 8 shows the impact process at different time instants. Figure 9 present the specimen after the impact process. As it can be seen two main damage can be observed: specimen erosion in the impacted face and delamination between plies. Also, some breakage between plies among the epoxy matrix can be seen. The water-slide paper used for the DIC slides detached once the breakage and delamination occur, which does not interfere in the different data obtained in the VIC-3D system.

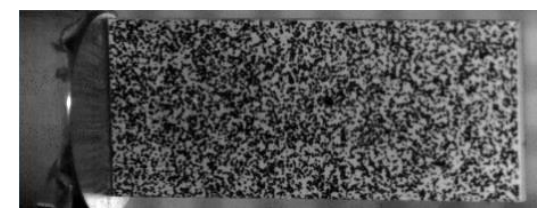

$t=0 \mathrm{~ms}$

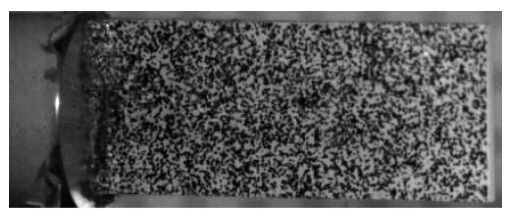

$t=0.05 \mathrm{~ms}$

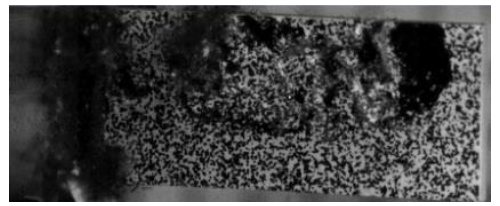

$t=0.2 \mathrm{~ms}$

Fig. 8. Impact experiment of CFRP launched at $113 \mathrm{~m} / \mathrm{s}$ 

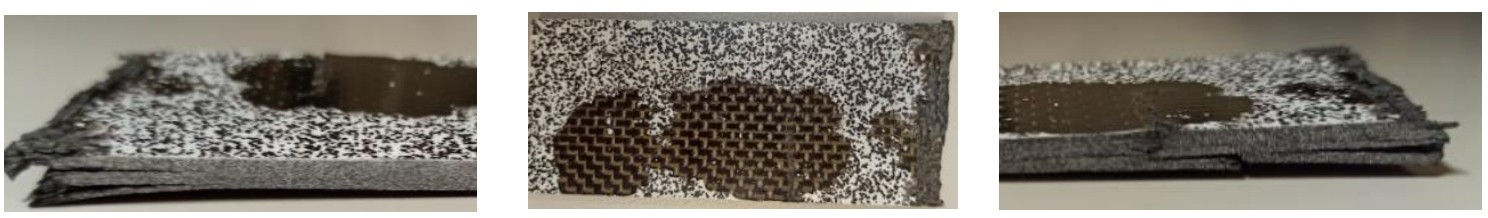

Fig. 9. Damage patterns of CFRP specimen at $113 \mathrm{~m} / \mathrm{s}$ a) Delamination b) Erosion c) Matrix Breakage

\section{Conclusions}

In this work the high velocity impact of Poly Methacrylate Methyl Amide (PMMA) and Carbon Fiber Reinforced Polymer (CFRP) have been analyzed against a Hopkinson Bar. Experimental tests have been performed using a gas gun while it has been recorded with 2 Photron SA-Z High Speed Cameras. The specimen compressive strains have been observed with the VIC-3D software, allowing to observe the strain field as the and an approximation to the failure strain. Semiconductor strain gages are implemented to increase the sensibility of the procedure to obtain the compressive pulses in the Hopkinson Bar, which are needed to analyze the impact force. The water-slide paper technique for the DIC have been successfully used. From the presented results, the main conclusions extracted are:

- The CFRP fragment presents an impact force almost 6 times higher than the PMMA fragment.

- Different compressive pulses have been observed in the CFRP fragment before failure, the two of them reaching a maximum compressive strain of $1.1 \%$.

- The damage pattern is very different comparing quasibrittle materials. PMMA fragments presents a totally breackage, while CFRP fragment shows different failure modes such as delamination, erosion, and matrix breackage.

\section{References}

[1] J. López-Puente, A. Mata-Díaz, J. Pernas-Sánchez, J.A. Artero-Guerrero, D. Varas. Experimental analysis of high velocity impacts of composite fragments, International Journal of impact Engineering 103 (2017), pp 231-240

[2] Laurence Vion et al., Counter-Rotating Open Rotor (CROR): flow physics and simulation, 20ème Congrès Français de Mécanique, 29 August-2 September 2011, Besançon, France(2011).

[3] J. López-Puente, A. Mata-Díaz, J. Pernas-Sánchez, J.A. Artero-Guerrero, D. Varas. Numerical study of composite fragment impacts onto rigid target, Composite Structures 203 (2018)

[4] Michael J. Pereira, Santo A. Padula, Santo A., Duane M. Revilock, Matthew E. Melis. NASA Technical Report. (2006)

[5] H. Koerber et al., High strain rate behaviour of 5-harness-satin weave fabric carbon-epoxy composite under compression and combined compression-shear loading, International Journal of Solids and Structures, 54 (2015), pp. 172-182

[6] J.Wang et al., The mechanical properties and constitutive model of two woven composites including the influences of temperature, strain rate and damage growth, Composites Part B, 161 (2019), pp 502-513.

[7] Gustavo Quino et al., Speckle patterns for DIC in challenging scenarios : rapid application and impact endurance, Measurement Science and Technology 32, n.o 1015203 (2020). 